\title{
ANÁLISIS DE LAS COMPETENCIAS NECESARIAS PARA UN BACHILLERATO GENERAL EN RECREACIÓN PARA COSTA RICA
}

\author{
Carmen Grace Salazar Salas \\ Escuela Educación Física y Deporte \\ Universidad de Costa Rica, San José, Costa Rica \\ E-mail: csalazar@cariari.ucr.ac.cr
}

\begin{abstract}
Resumen
Salazar Salas, C. G. (2002). Análisis de las competencias necesarias para un bachillerato general en recreación para Costa Rica. Revista de Ciencias del Ejercicio y la Salud, 2(2), 38-49. Los objetivos de este artículo son: 1. presentar los resultados de un estudio realizado para determinar el perfil profesional necesario para un bachillerato general en recreación de la Universidad de Costa Rica y 2. justificar la importancia de dicho bachillerato por medio de la revisión de literatura. El propósito del estudio fue determinar cuáles eran las competencias necesarias para el perfil profesional de un plan de estudios de un bachillerato general en recreación para que sea ofrecido por la Escuela de Educación Física y Deportes de la Universidad de Costa Rica. Para el estudio, se diseñó un cuestionario con 35 competencias del perfil profesional: 15 competencias de conocimiento, 12 de destreza, y ocho de actitud. Un total de 76 sujetos respondieron el cuestionario, distribuidos en cinco grupos: estudiantes y graduados universitarios, profesores universitarios, directores de programas recreativos privados, directores de parques nacionales, y personeros de la entonces Dirección General de Educación Física y Deportes, hoy Instituto Costarricense del Deporte y la Recreación. Una ANOVA de una vía mostró diferencias significativas en el nivel de importancia de cuatro de las 35 competencias entre los cinco grupos de sujetos. Además, todas las competencias obtuvieron promedios generales mayores que el número límite para ser tomadas en cuenta para el perfil en estudio. Por lo tanto, se concluyó que los encuestados consideraron a todas las competencias propuestas en el cuestionario necesarias para el perfil profesional de un bachillerato general en recreación. PALABRAS CLAVES: perfil profesional, competencias en recreación, profesión, bachillerato general, recreación.
\end{abstract}

\section{INTRODUCCIÓN}

"En Costa Rica, la recreación existe como actividad, pero no como profesión" (Salazar Salas, 1996, p. 63), cuya preparación en las aulas universitarias conlleve a la obtención de un grado académico. Es por ello que el campo de la recreación ha estado "a cargo" de los graduados de otras carreras; "pero, ya es necesario que se empiecen a formar profesionales en recreación, quienes, con la adquisición de competencias profesionales, proporcionen, programen, administren y conduzcan las actividades recreativas en el país" (Salazar Salas, p. 64). La preparación profesional en recreación debe iniciarse lo antes posible; pero también es vital que se divulgue qué es la recreación, pues la relevancia y el papel de este nuevo campo en la sociedad son poco conocidos a causa de la escasa información que existe acerca del tema.
Las vagas percepciones de lo que es recreación y su importancia en la sociedad son numerosas.

Los objetivos de este artículo son: 1 . presentar los resultados de un estudio realizado para determinar el perfil profesional necesario para un bachillerato general en recreación de la Universidad de Costa Rica y 2. justificar la importancia de dicho bachillerato por medio de la revisión de literatura.

\section{Definición de recreación}

La recreación es un conjunto de actividades placenteras realizadas durante el tiempo libre y que promueven el desarrollo integral de las personas. En la recreación, cada persona selecciona las actividades que desea realizar y, además, decide cuándo desea participar en ellas (Salazar Salas, 2003). La recreación es importante en la vida de cada 
persona y todos tienen derecho a participar en actividades recreativas. La participación en la recreación depende de una serie de factores intrínsicos y extrínsecos a los individuos; por lo tanto, los tipos de actividades recreativas son tan numerosos como los intereses y gustos de las personas. Las actividades recreativas se pueden realizar y disfrutar junto a otras personas, o de manera individual. La actividad puede demandar trabajo físico o mental, realizarse durante toda la vida, días, semanas o meses, o esporádicamente (Salazar Salas, 1996).

\section{Clasificación de actividades recreativas}

Para muchas personas, la recreación sólo incluye juegos, deportes, y paseos; por lo que no toman en cuenta otra gran cantidad de actividades recreativas. En una revisión de literatura acerca de este tema específico, se encontró que autores, tales como Farrell y Lundegren (1991), Kraus (1997), Russell (2002) y Stumbo (1992), incluyen las siguientes categorías en sus clasificaciones de actividades recreativas: 1. deportes, juegos y actividades físicas (actividades individuales, en parejas, en conjunto, entre otros), 2. pasatiempos (educativos, de colección, creativos, entre otros), 3. actividades culturales (música, drama, baile, artes plásticas), 4. recreación al aire libre y actividades relacionadas con la naturaleza (campamentos, jardinería, preservación de los recursos naturales, deportes al aire libre, entre otros), 5. actividades cognoscitivas (lectura, redacción, oratoria, estudio de temas, entre otros), 6. recreación social (fiestas, bailes, reuniones, visitas, conversaciones, entre otros), 7. turismo (en forma individual, en pequeños grupos, en grupos grandes), y 8 . actividades de enriquecimiento y actualización personal.

\section{Clasificación de formatos recreativos}

Los formatos recreativos consisten en las maneras en las que son organizadas y ofrecidas las actividades en las que pueden participar las personas. Autores como Edginton, Hanson, y Edginton (1992), Kraus, (1997), Russell (1982), y Rossman y Schlatter
(2000), proponen las siguientes categorías de formatos recreativos: 1 . clubes y grupos con intereses sociales, 2. competencias y concursos, 3. paseos, viajes y excursiones, 4. actividades o eventos especiales (actividades que se realizan durante varias horas o días, a veces en forma ocasional para celebrar alguna festividad), 5. instalación abierta (participar en un programa cuando se desea y cuando la instalación está abierta, como un gimnasio), 6 . trabajo voluntario, y 7 . clases, congresos, talleres, seminarios, y conferencias.

\section{Áreas de trabajo o ramas de la recreación}

La diversidad de programas que se pueden ofrecer en recreación se determina si se toman en cuenta las diferentes áreas de trabajo o ramas de la profesión. "Estas ramas se han establecido en los Estados Unidos a causa del interés de los profesionales, los investigadores y las publicaciones que se realizan" (Salazar Salas, 1996, p. 57). Varias de estas áreas de trabajo se traslapan pues tienen temas comunes (ver más detalles de estas áreas en Salazar Salas, 1996 y Kraus, 2001, 2003). Algunas de ellas son: (1) Recreación Comunitaria, (2) Recreación Comercial, (3) Recreación Terapéutica, (4) Recreación Universitaria, (5) Recreación al Aire Libre, (6) Recreación Laboral, y (7) Recreación Acuática.

\section{Factores que promueven la carrera de la recreación}

Desde 1990, Kraus identificó una serie de factores que han promovido el desarrollo de la recreación como profesión en otros países. Estos factores incluyen: (1) las personas disponen de mayor cantidad de tiempo libre, (2) las personas -algunas más que otrasdisponen de mayor cantidad de dinero para divertirse, (3) la participación en actividades recreativas ha aumentado a causa del incremento de la población y de su mejor nivel de educación (las personas con más alto nivel educativo valoran más los beneficios obtenidos por medio de la recreación), (4) existe mayor cantidad, variedad, y acceso a instalaciones recreativas, (5) existe mayor diversidad y comercialización de actividades recreativas, 
sobre todo las relacionadas con la tecnología, (6) el interés por las actividades artísticas ha aumentado, (7) las personas con alguna discapacidad y los adultos mayores tienen acceso a una mayor cantidad de actividades recreativas, (8) el reconocimiento de la importancia de la salud ha aumentado, (9) la mujer desempeña muchos y diversos puestos en la sociedad, y (10) la preocupación por preservar la naturaleza ha aumentado.

Beneficios que se pueden obtener con la participación en la recreación

Por sus efectos positivos, muchas personas consideran la recreación como una alternativa para resolver problemas sociales, pues este campo profesional contribuye a mejorar la calidad de vida de los ciudadanos (Salazar Salas, 1996). Se debe recordar que como la recreación vela por el desarrollo integral de las personas, trabaja en pro de los cuatro canales de desarrollo; sin embargo, enfoca un poco más en el canal psicológico. Si alguien está bien emocionalmente, eso es muestra de que los otros canales también lo están y de que la persona está saludable. Abramson (1996), Cadwell (1993), y Kraus (2001) manifiestan que diversas poblaciones que participan en programas recreativos experimentan tranquilidad, alegría, libertad de elección, motivación, e interacción social. Además, estos programas ayudan a los jóvenes a no involucrarse en grupos y actividades negativas y delictivas. Austin y Crawford (2001), Edginton, Hanson, y Edginton (1992), Kraus (2001), Mannell y Kleiber (1997), Parks and Recreation Federation of Ontario (1992), Sefton y Mummery (1995), y Witt y Crompton (1997) mencionan diferentes tipos de beneficios que se obtienen con la participación en actividades recreativas. Estos beneficios se pueden clasificar en: 1 . beneficios personales (físicos, psicológicos, sociales, intelectuales, y espirituales), y 2 . beneficios para la sociedad (de índole familiar, social, económico, y ambiental). Ver más detalles de la clasificación de los beneficios de la recreación en Salazar Salas $(1996,1997)$.

Importancia de la recreación y de sus propios profesionales en Costa Rica
La recreación es muy importante en la actualidad pues ella ofrece actividades que se pueden realizar durante el tiempo libre, el cual aumenta constantemente a causa de los avances tecnológicos. Si las personas no saben qué hacer durante ese tiempo, lo desaprovecharán o mal emplearán, y esas personas [podrían no sentirse] satisfechas ni contentas. La recreación [tiene como objetivo] el desarrollo integral de la persona. Si un individuo se siente realizado, será un mejor ciudadano y podrá contribuir [en el] bienestar de su hogar y del país. (Salazar Salas, 1996, p. 63)

Por estas razones es necesario que en Costa Rica exista una carrera específica en recreación que prepare su propio recurso humano, y no que sean los profesionales de otros campos, o personas empíricas, los que se hagan cargo de las actividades recreativas. Los profesionales en recreación estarían académicamente preparados para ofrecer una gran diversidad de programas recreativos para que los costarricenses hagan un buen uso de su tiempo libre (Kraus, 2001). Es importante que se inicie la formación de profesionales en recreación y se respete a este campo como a una nueva profesión $\mathrm{y}$, sobre todo, independiente de la educación física, carrera de la cual aún muchas personas la consideran parte.

Los profesionales en recreación deben probarle a la sociedad la relevancia y el valor que su campo de trabajo tiene, y la necesidad y la importancia de su preparación profesional específica. "Un profesional en recreación es quien [-con conocimiento de la filosofía de la recreación como carrera-] programa actividades recreativas para distintas poblaciones, y quien utiliza conocimientos administrativos para lograr el éxito de tales actividades" (Salazar Salas, 1996, p. 61). Estos programas recreativos pueden beneficiar a una gran gama de grupos. Los programas se pueden ofrecer a poblaciones específicas o a varias en conjunto. Algunas de estas poblaciones incluyen: niños, jóvenes, adultos, adultos mayores, estudiantes, trabajadores, mujeres, hombres, y personas con alguna discapacidad, entre otras. En un programa 
recreativo, existe la posibilidad de que el profesional en recreación ocupe cuatro puestos: instructor/ líder, programador, supervisor, y administrador. En otros países, gran parte de estos profesionales se desempeñan en uno de estos puestos cuando el programa recreativo a cargo es muy grande. $\mathrm{Si}$ el programa es pequeño, él permite que un mismo profesional desempeñe simultáneamente los cuatro puestos o varios de ellos. Si el profesional desea enseñar, debe estar capacitado académicamente para hacerlo. No obstante lo anterior, se debe tener claro que no sólo los profesionales en recreación pueden trabajar en programas recreativos. Los profesionales en danza, educación física, música y muchas otras profesiones pueden ser instructores en cursos o líderes en programas recreativos diseñados por profesionales en recreación (Russell, 2001; Salazar Salas, 1996; Southwest Texas State University, s.f.).

La apertura de la carrera en recreación en Costa Rica es necesaria por seis razones. Primero, se necesita personal capacitado en recreación. Son pocos los costarricenses que poseen un título en recreación: títulos que obtuvieron en el extranjero. Estos profesionales constituyen una fuente importante para capacitar a otras personas interesadas en obtener una preparación académica propia en recreación. Si en Costa Rica se ofreciera la carrera en recreación, muchos costarricenses tendrían la oportunidad de estudiar esta profesión en el país sin tener que trasladarse al extranjero, como hasta ahora ha ocurrido.

Segundo, se necesita profesionales preparados que, con las competencias específicas del campo, sean capaces de velar y poner en práctica la filosofía de la recreación por medio de actividades recreativas. La filosofía de la recreación consiste en mejorar la calidad de vida de los participantes de sus programas. En recreación, la principal función de los profesionales es la de programación. Por eso, el profesional en recreación debe estar bien preparado en esta área. Sin embargo, también debe estudiar otros temas tales como instalaciones deportivas, recursos humanos, las diferentes áreas de trabajo de la recreación y sus características, entre otros (Kraus, 2001; Russell, 2001; Salazar Salas, 1996).

Tercero, sólo teniendo más profesionales en recreación, la sociedad estaría segura de que podría disfrutar de un mayor repertorio de actividades recreativas que satisfagan sus intereses y necesidades, y que contribuyan a mejorar su salud (Salazar Salas, 1993). En este momento en el que la salud de los ciudadanos es tema prioritario, se debe considerar a la recreación como una de las carreras que podría contribuir sustancialmente en la promoción de la salud, ya que sus programas se desarrollan dentro del sistema no formal de enseñanza. Por ejemplo, gran cantidad de personas de diferentes edades y estratos sociales participa en programas de ejercicio preventivo en gimnasios (Ho, Payne, Orsega-Smith, y Godbey, 2003).

Cuarto, la cantidad de tiempo libre y la población han aumentado en los últimos años. Por lo tanto, se necesita personal preparado para promover el buen uso de ese tiempo por medio de actividades recreativas y la valorización de los beneficios que la recreación le proporciona a quienes participan en ella.

Quinto, la cantidad de instalaciones y programas recreativos en las comunidades han aumentado y se necesita profesionales que los administren, organicen, conduzcan y evalúen.

Sexto, se necesita personal especializado en este campo que se integre plenamente en la generación y la actualización de políticas nacionales acerca de la recreación, y en la asesoría de temas recreativos a organizaciones gubernamentales, privadas, $\mathrm{y}$ voluntarias.

\section{Perfil profesional de recreación}

Ya que en Costa Rica se hace imperiosa la preparación de profesionales en el campo de la recreación, es necesario tener un perfil profesional que guíe tal currículo, y como hasta 1993 no se había identificado ninguno, a continuación se detalla un estudio, tema de una tesis de maestría en recreación de la Universidad del Sur de Illinois (Salazar Salas, 1993; Salazar Salas y McEwen, 1996), que se abocó a determinar el perfil profesional 
para la carrera de un bachillerato general en recreación para Costa Rica.

Pero, ¿qué es un perfil profesional? Según el Centro de Evaluación Académica de la Vicerrectoría de Docencia de la Universidad de Costa Rica, un perfil de este tipo es el "conjunto de características que debe presentar un profesional en términos de conocimientos, cualidades personales, habilidades y actitudes y valores necesarios para ejercer adecuadamente las funciones en el campo de su especialidad" (Vargas Porras, 1992, p. 17). Por su parte, las características o competencias profesionales son definidas por el Centro de Evaluación Académica como "saberes científicos (teóricos y prácticos, conceptuales y valóricos) y populares adquiridos por los universitarios, en el mundo académico y el mundo de la vida cotidiana respectivamente, que les permiten el desempeño adecuado de una profesión" (Universidad de Costa Rica, 1999, p. 99). Las competencias de conocimiento incluyen la "información requerida para la ejecución de las responsabilidades; comprenden conceptos, hechos y criterios" (Vargas Porras, 1992, p. 17). Las competencias de habilidad o destreza se refieren a las "capacidades motoras y/o cognoscitivas necesarias para realizar la actividad descrita por una responsabilidad [laboral]" (Vargas Porras, p. 17). Las competencias de actitud se basan en la "disposición para actuar al realizar una actividad. Comprenden valores, sentimientos y otros aspectos afectivos" (Universidad de Costa Rica, 1991, p. 53).

\section{Problema del estudio}

Con la finalidad de determinar un perfil profesional en recreación, el problema de este estudio consistió en: ¿Cuáles son las competencias de conocimiento, de destreza y de actitud necesarias para el perfil profesional de un plan de estudios de un bachillerato general en recreación para la Escuela de Educación Física y Deportes de la Universidad de Costa Rica?

\section{METODOLOGIA}

\section{Sujetos}

En el estudio se consideraron cinco grupos de sujetos con base en su experiencia e interés en el campo de la recreación. En total, los sujetos fueron: (a) 18 profesores de la Escuela de Educación Física y Deportes de la Universidad de Costa Rica y de la Escuela de Ciencias del Deporte de la Universidad Nacional. Se les escogió porque muchos de ellos han estado involucrados en programas recreativos, y han participado en la elaboración de planes de estudio, por lo que tienen experiencia en determinar perfiles profesionales; (b) 23 estudiantes y graduados del entonces énfasis de Administración Deportiva y Recreativa de la carrera de Bachillerato en Educación Física de la Universidad de Costa Rica. Ellos poseen y ponen en práctica diversas competencias del perfil y con su experiencia se han percatado del perfil que un profesional en recreación necesita; (c) 18 directores de programas recreativos privados. Al conducir programas recreativos y estar en contacto con la gente, ellos han podido identificar algunas de las competencias necesarias en el campo y las demandas de la recreación comercial; (d) 11 directores de parques nacionales. Con su experiencia en la recreación al aire libre, ellos han podido detectar algunas posibles competencias del perfil profesional en recreación; y (e) seis miembros de la entonces Dirección General de Educación Física y Deportes (DEFYD), hoy Instituto Costarricense del Deporte y la Recreación (ICODER). Al trabajar en una institución pública que ofrece programas de recreación comunitaria, ellos conocen las políticas del gobierno en cuanto a la recreación y tienen experiencia en la elaboración y puesta en práctica de programas recreativos.

\section{Instrumento de medición}

La información para la investigación se recolectó por medio de un cuestionario. Para confeccionar el instrumento, se realizó una revisión de literatura relacionada con la 
recreación, con el fin de obtener una lista de competencias útiles para el perfil profesional en estudio.

Para seleccionar las competencias de conocimiento y de destreza, se revisaron seis fuentes de información: Austin (1985), Cipriano (1984), Kelly, Robb, Park y Halberg (1976), McLellan y Pope (1984), National Recreation and Park Association (NRPA) (1990), y Priest (1989). Se identificaron 431 competencias. Algunas competencias estándares de la NRPA se usaron como base para compararlas con otras. Para las competencias de actitud, se usaron cinco fuentes: Austin (1985), Escuela Educación Física y Deportes (s.f.), Henderson y Bedini (1989), Priest (1989), y Vargas-Porras (1992). Se encontraron 38 competencias de actitud.

Del total de 469 competencias encontradas, algunas se excluyeron porque no correspondían a un perfil de recreación general, que era el objetivo del estudio, pues se relacionaban más con Estudios Generales, Recreación al Aire Libre, y Recreación Terapéutica. Muchas de las competencias fueron unidas, por lo que, el primer borrador incluyó un total de 75 competencias. Después de una nueva revisión, se conservaron 34 competencias.

Con estas 34 competencias, se confeccionó un instrumento que tenía tres partes: la carta de presentación del estudio; tres secciones, una por grupo de competencias; y un espacio para agregar comentarios al cuestionario. Se realizó una prueba piloto del cuestionario borrador. Se tradujo al español y se envió a algunos profesores de la Escuela de Educación Física y Deportes de la Universidad de Costa Rica, a principios de marzo de 1993. A ellos se les pidió que revisaran las competencias seleccionadas, recomendaran otras que se hubieran omitido, e hicieran sugerencias para mejorar el cuestionario. Todos estos comentarios fueron recibidos a principios de abril de 1993. Dentro de los comentarios se recomendó cambiarle la redacción a algunas competencias, sustituir algunas palabras por otras más comunes, y dividir una competencia en dos.

Como resultado de la prueba piloto y las recomendaciones de la Comisión de Tesis, la versión final del instrumento consistió en la carta de presentación del estudio y en 35 competencias. Las 35 competencias se mantuvieron en tres secciones. La primera tenía 15 competencias de conocimiento; la segunda, 12 competencias de destreza; y la tercera, ocho competencias de actitud. En cada sección se le pidió a los encuestados que, con base en su experiencia y conocimiento y usando una escala de cinco puntos, clasificaran la importancia de las 35 competencias de ser parte de un bachillerato general ene recreación. Esta escala incluía los criterios: Esencial (valor cinco puntos), Muy Importante, Importante, Poco Importante, y No Importante (valor un punto). Ver el instrumento completo en Salazar Salas (1993).

\section{Procedimientos}

A finales de mayo de 1993, se envió 100 copias del cuestionario a Costa Rica. La recolección de datos duró casi tres meses. Como la investigadora no estaba en el país, la persona a cargo de la administración del cuestionario entregó las copias necesarias a otras personas, quienes a su vez lo administraron a varios grupos de personas. El cuestionario fue entregado a aquellos sujetos que aceptaron voluntariamente ser parte del estudio. Se trató de localizar al mayor número posible de personas. Como las instrucciones estaban en el cuestionario, no se requerían explicaciones extra. Sólo se debía leer la carta y llenar el cuestionario.

En la Escuela de Educación Física y Deportes de la Universidad de Costa Rica, el cuestionario fue distribuido entre los docentes por la Directora de la Escuela. Una docente que trabajaba en esta Universidad y en la Universidad Nacional llevó las copias del cuestionario a los profesores de la Escuela de Ciencias del Deporte de la Universidad Nacional. Al final, 18 profesores de ambas Universidades completaron el cuestionario. Con respecto a los estudiantes activos del énfasis de Administración Deportiva y Recreativa del Bachillerato en Educación Física de la Universidad de Costa Rica, ellos contestaron el cuestionario en clase. A los graduados del énfasis se les consultó por 
teléfono si deseaban formar parte del estudio. El administrador del cuestionario visitó los lugares de trabajo de estas personas para facilitarles el cuestionario. En total, 23 estudiantes y egresados respondieron el instrumento.

Los directores de parques nacionales se localizaron por medio de uno de ellos, quien les dio los cuestionarios a sus compañeros en una reunión de directores. Once directores de parques nacionales contestaron el cuestionario. Dieciocho directores de programas recreativos privados contestaron el cuestionario en sus trabajos, agencias de turismo. El cuestionario fue dejado en tales lugares y luego recogido. Seis miembros de la Sección de Recreación de la DEFYD contestaron el cuestionario en su trabajo. El cuestionario también les fue llevado a tal institución.

Para efectos de determinar cuales competencias debían incluirse en el perfil profesional de un bachillerato general en recreación, al inicio del estudio se preestableció que todas aquellas competencias cuyo promedio general fuera igual o mayor que 3 serían tomadas en cuenta para conformar el perfil. El número 3 equivalía al nivel de Importante en la escala del instrumento del estudio. Las competencias con un promedio general inferior a 3, no debían ser incluidas en el perfil.

\section{Análisis estadístico}

Los datos se codificaron y analizaron con el programa estadístico SAS. En la estadística descriptiva se utilizaron frecuencias, porcentajes, y promedios. En la estadística inferencial se empleó ANOVA.

\section{RESULTADOS}

\section{Estadística descriptiva}

Se usó estadística descriptiva para resumir y clasificar información. Para efectos de facilitar el análisis y el reporte de los resultados en este artículo, algunas palabras de cada competencia fueron usadas como el nombre de las competencias. Por ejemplo, Conocer diagnóstico necesidades recreativas equivale a la competencia de conocimiento Conocer los procedimientos y técnicas para diagnosticar las necesidades recreativas de los costarricenses.

\section{Frecuencias y porcentajes en cada nivel} de la escala del cuestionario

La Tabla 1 muestra las frecuencias y los porcentajes obtenidos en las respuestas dadas en cada nivel de la escala usada en el cuestionario, en cada uno de los grupos de competencias. Esta tabla también incluye los "porcentajes promedio" en cada uno de los tres grupos de competencias. La categoría correspondiente a un punto (No importante) obtuvo un porcentaje promedio de $0.7 \%$ en los tres grupos de competencias; mientras que la categoría de dos puntos (Poco Importante) obtuvo un porcentaje promedio en los tres grupos de competencias de $4.6 \%$. La categoría de los tres puntos (Importante) obtuvo un porcentaje promedio entre los tres grupos de competencias de $25.4 \%$. Las categorías con los porcentajes promedio más altos entre los tres grupos de competencias fueron las categorías de cuatro puntos (Muy Importante) y de cinco puntos (Esencial) con 35.8\% y 33.2\%, respectivamente. En resumen, más del $66 \%$ de las respuestas dadas por los entrevistados se concentraron en los niveles 4 (Muy Importante) y 5 (Esencial). Un $25 \%$ de las respuestas fueron para el nivel 3 (Importante), y menos del $6 \%$ fueron para los niveles 2 (Poco Importante) y 1 (No Importante).

\section{Promedios en cada grupo de competencias}

\section{Competencias de conocimiento}

En las competencias de conocimiento, la competencia Conocer programación actividades obtuvo el promedio más alto, 4.50. Por el contrario, el promedio más bajo, 3.26, le correspondió a la competencia Conocer conceptos legales. De las 15 competencias de conocimiento, ocho (53\%) obtuvieron un promedio mayor que 4, por lo que los sujetos las consideraron Muy Importantes. Las otras siete $(47 \%)$ competencias fueron consideradas Importantes al obtener un promedio mayor que 3 , pero menor que 4 . 
Tabla 1. Frecuencias y Porcentajes en las Respuestas Dadas en Cada Nivel de la Escala del Cuestionario

\begin{tabular}{|c|c|c|c|c|c|c|c|c|c|c|c|c|c|}
\hline \multirow{3}{*}{$\begin{array}{c}\text { Grupo de } \\
\text { competencias }\end{array}$} & \multicolumn{10}{|c|}{ Niveles de la Escala del Cuestionario } & & & \multirow{3}{*}{ Total } \\
\hline & \multicolumn{2}{|c|}{$\begin{array}{c}1 \\
\text { No Importante }\end{array}$} & \multicolumn{2}{|c|}{$\begin{array}{c}2 \\
\text { Poco } \\
\text { Importante }\end{array}$} & \multicolumn{2}{|c|}{$\begin{array}{c}3 \\
\text { Importante }\end{array}$} & \multicolumn{2}{|c|}{$\begin{array}{c}4 \\
\text { Muy } \\
\text { importante }\end{array}$} & \multicolumn{2}{|c|}{$\begin{array}{c}5 \\
\text { Esencial }\end{array}$} & \multicolumn{2}{|c|}{$\begin{array}{c}\text { No } \\
\text { contestaron }\end{array}$} & \\
\hline & $n$ & $\%$ & $n$ & $\%$ & $n$ & $\%$ & $n$ & $\%$ & $n$ & $\%$ & $n$ & $\%$ & \\
\hline Conocimiento & 10 & 0.8 & 61 & 5.4 & 296 & 26 & 387 & 34 & 383 & 33.6 & 3 & 0.2 & 1140 \\
\hline Destrezas & 7 & 0.8 & 53 & 5.8 & 286 & 31.4 & 342 & 37.5 & 220 & 24.1 & 4 & 0.4 & 912 \\
\hline Actitud & 2 & 0.3 & 8 & 1.3 & 95 & 15.6 & 224 & 36.8 & 279 & 46 & 0 & 0 & 608 \\
\hline TOTAL & 19 & & 122 & & 677 & & 953 & & 882 & & 7 & & 2660 \\
\hline $\begin{array}{l}\text { Porcentaje } \\
\text { Promedio }\end{array}$ & & 0.7 & & 4.6 & & 25.4 & & 35.8 & & 33.2 & & 0.3 & \\
\hline
\end{tabular}

Competencias de destreza

La competencia de destreza con el promedio más alto, 4.34, fue Diagnosticar necesidades recreativas. La competencia con el promedio más bajo, 3.10, fue Aplicar conceptos legales. De las 12 competencias de destreza, cinco $(42 \%)$ obtuvieron un promedio mayor que 4; por lo que fueron consideradas como Muy Importantes. Las otras siete (58\%) competencias fueron consideradas Importantes al tener un promedio mayor que 3 y menor que 4.

\section{Competencias de actitud}

La competencia de actitud Mostrar entusiasmo profesional obtuvo el promedio más alto, 4.56; mientras que Mostrar características personales saludables obtuvo el promedio más bajo, 3.96. De las ocho competencias de actitud, siete (88\%) obtuvieron un promedio mayor que 4 , lo que implica que la mayoría de estas competencias fueron consideradas Muy Importantes para el perfil profesional.

\section{Promedios de todas las competencias}

De las 35 competencias, la competencia de actitud, Mostrar entusiasmo profesional obtuvo el promedio más alto, 4.56; y la competencia de destreza Aplicar conceptos legales el promedio más bajo, 3.10. Veinte $(57 \%)$ de las 35 competencias fueron consideradas como Muy Importantes al obtener un promedio mayor que 4, y 15 (43\%) competencias fueron clasificadas como Importantes al obtener promedios mayores que 3 y menores que 4 .

\section{Estadística inferencial}

ANOVA por competencia

Para establecer si había diferencia significativa entre los promedios de cada competencia entre los cinco grupos de sujetos, se realizaron 35 ANOVAs con $\mathrm{n}$ diferentes, una ANOVA por competencia. A causa del gran número de análisis realizados, el error Tipo I (rechazar la hipótesis nula cuando es verdadera) se incrementó. Para controlar este error, se efectuó la corrección Bonferroni. Este procedimiento consiste en dividir el alpha original (0.05) entre el número de tests efectuados (35). El nuevo alpha fue de 0.0014. Los resultados mostraron que sólo cuatro competencias eran estadísticamente significantes. Estas competencias fueron Conocer técnicas de liderazgo (F: 8.39, pF: $0.0001)$; Conocer organización comunal (F: 6.84, pF: 0.0001); Evaluar programas y servicios recreativos (F: 5.21, pF: 0.0010); y Reconocer necesidad de actualización en el campo (F: 7.53, pF: 0.0001).

Con la finalidad de detectar el origen de la diferencia de criterios acerca de la importancia de estas cuatro competencias, la Tabla 2 muestra los promedios de cada una de las cuatro competencias en los cinco grupos de encuestados. Como se puede apreciar en esta tabla, uno de los dos grupos que más discrepancias mostró en los promedios de las cuatro competencias con diferencia significativa fue el de directores de programas recreativos privados. Tres de sus cuatro promedios fueron los más bajos. Por otro lado, los personeros de la DEFYD se caracterizaron 
porque tres de sus cuatro promedios fueron los más altos.

Tres de los promedios de los directores de parques fueron un poco bajos. Los estudiantes y graduados, y los profesores se caracterizaron porque tres de sus promedios eran intermedios.

\section{DISCUSION}

Al comparar promedios entre las competencias de conocimiento y las de destreza, se notó que los encuestados costarricenses consideraron los temas relacionados con "la programación de actividades recreativas", "el diseño de instalaciones", y "el diagnóstico de necesidades recreativas de los participantes" como muy importantes. Las competencias relacionadas con estos temas fueron clasificadas en los primeros lugares de los promedios de cada uno de estos dos tipos de competencias.

En Costa Rica, el determinar las necesidades recreativas casi no se realiza; por lo tanto, la programación de servicios no satisface, en ocasiones, las expectativas de los participantes. Estos promedios podrían reflejar el reconocimiento de los encuestados acerca de la necesidad de incluir estas dos competencias en el perfil de recreación para proporcionar una preparación adecuada a los profesionales en el campo.

Los encuestados no consideraron las competencias de los temas concernientes a "la elaboración de presupuestos" y a "los conceptos legales", las dos competencias de conocimiento y de destreza con promedios generales más bajos, tan importantes como el resto de competencias. La razón para esto podría ser que en Costa Rica las personas no están acostumbradas a demandar a otras por lesionarse en actividades recreativas. Además, los costarricenses podrían no estar conscientes de que administrar instalaciones y ofrecer programas recreativos requieren un presupuesto formal para trabajar apropiadamente. Los encuestados podrían no haber tenido claro lo que la formación profesional de la carrera en recreación implica y requiere en cuanto a presupuesto se refiere.
Es interesante notar que Mostrar entusiasmo profesional, una competencia de actitud, tuvo el promedio más alto de las 35 competencias. Esto podría indicar que los encuestados percibieron esta característica como un elemento fundamental para un recreacionista porque él o ella tiene que ser entusiasta para incentivar a las personas a participar en actividades recreativas.

A pesar de que las 35 competencias obtuvieron promedios generales mayores que 3 , y -por lo tanto- fueron consideradas importantes para ser parte del perfil para un bachillerato general en recreación, los resultados de la ANOVA mostraron que los cinco grupos de encuestados no coincidieron en el nivel de importancia de las competencias de conocimiento Conocer técnicas de liderazgo y Conocer organización comunal, la competencia de destreza Evaluar programas y servicios recreativos, y la competencia de actitud Reconocer necesidad de actualización en el campo. Los directores de programas recreativos privados y los personeros de la DEFYD fueron los encuestados que influyeron más en los promedios generales con sus clasificaciones acerca de la importancia de las competencias para ser parte de un bachillerato general en recreación.

\section{CONCLUSIONES}

\section{A. Con base en los resultados de este estudio, se concluye:}

1. La mayoría de los encuestados consideraron a las 35 competencias del perfil profesional propuesto como importantes, muy importantes, o esenciales al clasificarlas con puntajes de 3,4 ó 5 , según la escala del cuestionario. Los puntajes 1 (No Importante) y 2 (Poco Importante) fueron usados por menos del $6 \%$ de los encuestados. Un total de 15 de las 35 competencias tuvieron promedios entre 3 y 4 y el resto de competencias tuvo promedios entre 4 y 5 . 
Tabla 2. Promedios de cada una de las cuatro competencias en las que hubo diferencia significativa entre los cinco grupos de encuestados

\begin{tabular}{|c|c|c|c|c|c|}
\hline \multirow[b]{2}{*}{ Competencias } & \multicolumn{5}{|c|}{ Grupos de Encuestados } \\
\hline & $\begin{array}{c}\text { Estudiantes y } \\
\text { Graduados }\end{array}$ & $\begin{array}{c}\text { Profesores } \\
\text { Universitarios }\end{array}$ & $\begin{array}{c}\text { Directores de } \\
\text { programas } \\
\text { recreativos privados }\end{array}$ & $\begin{array}{c}\text { Directores de } \\
\text { parques } \\
\text { nacionales } \\
\end{array}$ & $\begin{array}{c}\text { Personeros } \\
\text { de la } \\
\text { DEFYD }\end{array}$ \\
\hline $\begin{array}{l}\text { Conocer técnicas de } \\
\text { liderazgo }\end{array}$ & 4.52 & 4.5 & 3.39 & 3.73 & 5.00 \\
\hline $\begin{array}{l}\text { Conocer organización } \\
\text { comunal }\end{array}$ & 3.30 & 4.17 & 2.94 & 3.73 & 4.17 \\
\hline $\begin{array}{l}\text { Evaluar programas y } \\
\text { servicios recreativos }\end{array}$ & 4.26 & 4.28 & 3.50 & 4.00 & 4.83 \\
\hline $\begin{array}{l}\text { Reconocer necesidad de } \\
\text { actualización en el } \\
\text { campo }\end{array}$ & 4.87 & 4.17 & 4.50 & 4.27 & 4.17 \\
\hline
\end{tabular}

2. Las competencias de conocimiento y de destreza con promedios más altos coincidieron en los mismos temas: "diseño, organización y conducción de programas recreativos", "diseño de instalaciones", y "la determinación de necesidades recreativas". Esto podría implicar que los participantes del estudio percibieron estas competencias como una prioridad en la formación de los recreacionistas y parte fundamental en un plan de estudios para un bachillerato general en recreación.

3. Los encuestados consideraron que los temas referentes a "presupuesto" y "conceptos administrativos legales" no eran tan importantes como otras competencias del estudio. Estos temas tuvieron los promedios más bajos como competencias de conocimiento y de destreza. Esto no significa que los encuestados hubieran percibido estos temas como irrelevantes porque los promedios fueron mayores que 3 . Quizá, los participantes del estudio pensaron que había otras competencias más relevantes para un plan de estudios en recreación. Siendo consciente de que en Costa Rica no hay un plan de estudios en recreación $\mathrm{y}$ que existen muchos temas desconocidos y no usados que deberían ser parte de un currículo de este tipo, se podría deducir que los sujetos desconocían la relevancia de las competencias Conocer y Usar los conceptos legales y Conocer y Usar los presupuestos en la administración de instalaciones y programas recreativos. Esta falta de conocimiento o información pudo haber influenciado en los bajos promedios otorgados a las competencias de estos dos temas.

4. Mostrar entusiasmo profesional, una competencia de actitud, fue considerada como la competencia más importante al obtener el promedio más alto de las competencias de actitud e inclusive de las 35 competencias.

5. A pesar de los diferentes bagajes académicos, experiencias y ocupaciones, todos los encuestados estuvieron de acuerdo en que las 35 competencias del estudio proporcionaban una base sólida para el perfil profesional de un plan de estudios de un bachillerato general en recreación, pues los promedios generales lo indicaron así y porque, según la ANOVA, los encuestados no concordaron en el nivel de importancia de cuatro de las 35 competencias para ser parte del perfil en estudio.

6. Las competencias empleadas en el instrumento y consideradas por los encuestados como importantes para un 
perfil de recreación general confirman la validez de las competencias y áreas investigadas o propuestas por Austin (1985), Cipriano (1984), Escuela Educación Física y Deportes (s.f.), Henderson y Bedini (1989), Kelly, Robb, Park y Halberg (1976), McLellan y Pope (1984), National Recreation and Park Association (NRPA) (1990), Priest (1989), y Vargas-Porras (1992).

\section{Acerca de la apertura de un bachillerato en} recreación

El país realmente necesita que se ofrezca un bachillerato en recreación que forme sus propios profesionales. Profesionales con competencias específicas de la carrera, quienes aprendan los temas básicos durante su formación universitaria, y no que haya personas empíricas que aprendan haciendo mientras experimentan sin ningún respaldo teórico. El ofrecer esta nueva carrera conllevará a iniciar muchos programas recreativos bien planificados no existentes, sobre todo a ciertas poblaciones necesitadas de ellos.

Una carrera en recreación tiene la característica de ofrecer trabajo no sólo a los recreacionistas, sino a un sinnúmero de profesionales en otros campos. Se debe recordar que no todos los programas recreativos tienen la peculiaridad de que la misma persona los programe, administre, y conduzca.

Al existir una carrera en recreación, sus profesionales podrían ayudar a mejorar la calidad de vida de los costarricenses al promover un equilibrio entre el trabajo y la recreación, la valorización de las actividades recreativas, y el buen uso del tiempo libre.

\section{REFERENCIAS}

Abramson, J. (1996). Put money where your mouth is: City backs recreation. Parks and Recreation, 31(3), 64-76.

Austin, D. R. (1985). Professional leadership. Competencies required for special recreation programs. Journal of Physical Education, Recreation and Dance, 56(5), 26-28.
Austin, D. R. y Crawford, M. E. (2001). Therapeutic recreation. An introduction (3era. ed.). Needham Heights, MA: Allyn and Bacon.

Cadwell, L. L. (1993). Research on adolescents and leisure activities. Parks and Recreation, 28(3), 19$21,23,25,161$.

Cipriano, R. E. (1984). Curriculum development. A task analysis approach. Journal of Physical Education, Recreation and Dance, 55(7), 55-51.

Edginton, C. R., Hanson, C. J. y Edginton, S. R. (1992). Leisure programming. Concepts, trends, and professional practice (2da. ed.). Dubuque, IA: Brown \& Benchmark

Escuela de Educación Física y Deportes (s.f.). Perfil general del bachiller de Educación Física. San José: Escuela Educación Física y Deportes.

Farrell, P. y Lundegren, H. M. (1991). The process of recreation programming. Theory and technique (3ra. ed.). State College, PA: Venture Publishing, Inc.

Henderson, K. A. \& Bedini, L. A. (1989). Teaching ethics and social responsibility in leisure studies curricula. SCHOLE: A Journal of Leisure Studies and Recreation Education, 4, 1-13.

Ho, C.-H., Payne, L., Orsega-Smith, E., y Godbey, G. (2003). Parks, recreation and public health. Parks and Recreation, 38(4), 18, 20-27.

Kelly, J. D., Robb, G. M., Park, W., \& Halberg, K. J. (1976). Therapeutic recreation education:Development a competency-based entrylevel curriculum. Urbana, Champaign: University of Illinois.

Kraus, R. (1990). Recreation and leisure in modern society (4ta. ed.). HarperCollins Publishers.

Kraus, R. (1997). Recreation programming. A benefitdriven approach. Needham Heights, MA: Allyn and Bacon

Kraus, R. (2001). Recreation and leisure in modern society (6ta. ed.). Sudbury, MA: Jones and Bartlett.

Kraus, R. (2003). Careers in recreation. Expanding horizons. Parks and Recreation, 73(5), 46-49, 54.

McLellan, R. W., \& Pope, J. R. Jr. (1984). Intramuralrecreation programs. Selecting qualified coordinators. Journal of Physical Education, Recreation and Dance, 55(6), 57-58-83.

Mannell, R. C. y Kleiber, D. A. (1997). A social psychology of leisure. State College, PA: Venture Publishing, Inc.

National Recreation and Park Association/Council on Accreditation. (1990). Standards and evaluative criteria for recreation, park resources and leisure services baccalaureate program. National Recreation and Park Association.

Parks and Recreation Federation of Ontario. (1992). The benefits of parks and recreation. Ontario, Canadá: Parks and Recreation Federation of Ontario y Ontario Ministry of Tourism and Recreation.

Priest, S. (1989). International experts rank critical outdoor leadership concerns and components. Journal of Physical Education, Recreation and Dance, 60(2), 72-77. 
Rossman, J. R. y Schlatter, B. E. (2000). Recreation programming. Designing leisure experiences (3ra. ed.). Sagamore Publishing.

Russell, R. V. (1982). Planning programs in recreation. Saint Louis: The C.V. Mosby Company.

Russell, R. V. (2001). Leadership in recreation (2da.ed.). NY: McGraw Hill.

Russell, R. V. (2002). Pastimes. The context of contemporary leisure (2da.ed.). Champaign, IL: Sagamore Publishing.

Salazar Salas, C. G. (1993). Competencies needed for an undergraduate general recreation curriculum for the University of Costa Rica. Tesis de maestría sin publicar, Southern Illinois University, Carbondale, Illinois, Estados Unidos.

Salazar Salas, C. G. (1996). Educación física y recreación. Dos carreras diferentes, pero complementarias. Educación, 20(1), 57-65.

Salazar Salas, C. G. (1997). Educación para el uso del tiempo libre. Educación, 21(2), 51-63.

Salazar Salas, C. G. (2003). Definiciones y conceptos. Curso Recreación y Juegos Organizados. Escuela Educación Física y Deportes, Universidad de Costa Rica. San José, Costa Rica: la autora.

Salazar Salas, C. G. y McEwen, D. N. (1996). Perceptions of Costa Rican professionals and students on the importance of the United States undergraduate recreation curriculum competencies for Costa Rica. Schole, 11, 73-83.

Sefton, J. M. y Mummery, W. R. (1995). Benefits of recreation research update. Pennsylvania: Venture Publishing, Inc.

Southwest Texas State University, Department of Health, Physical Education and Recreation. (s.f.). Agency application for site approval. San Marcos, Texas: el autor.

Stumbo, N. J. (1992). Leisure education II: More activities and resources. State College, PA: Venture Publishing, Inc.

Universidad de Costa Rica. Vicerrectoría de Docencia. Centro de Evaluación Académica. (1991). Guía para la elaboración y presentación de modificaciones a planes de estudios. San José, Costa Rica: Oficina de Publicaciones de la Universidad de Costa Rica.

Universidad de Costa Rica. Vicerrectoría de Docencia. Centro de Evaluación Académica. (1999). El proceso de autoevaluación para la acreditación. Una guía orientadora. San José, Costa Rica: Oficina de Publicaciones de la Universidad de Costa Rica.

Vargas Porras, A. E. (1992). Lineamientos para la elaboración de perfiles profesionales. San José: Oficina de Publicaciones de la Universidad de Costa Rica.

Witt, P. A. y Crompton, J. L. (1997). The at-risk youth recreation project. Parks and Recreation, 32(1), 5461. 